\title{
Metodologías y materiales para el aprendizaje de la anatomía humana. Percepciones de los estudiantes de medicina 'nativos digitales'
}

\author{
Blanca Mompeó-Corredera
}

Objetivo. Conocer la opinión de los estudiantes de la primera generación que ha crecido bajo el imperio de las tecnologías de la información y comunicación sobre las metodologías utilizadas actualmente en el proceso de enseñanza-aprendizaje de la anatomía humana en los estudios de medicina.

Sujetos y métodos. Se realiza un estudio a partir de los datos aportados por una encuesta contestada anónimamente por 74 estudiantes de segundo curso de la titulación de medicina en la Universidad de Las Palmas de Gran Canaria.

Resultados y conclusiones. En general, las metodologías mejor consideradas para el aprendizaje de la anatomía humana fueron el estudio de casos y el reconocimiento de estructuras en material humano fijado (prosecciones y disección), siendo la principal ventaja de esta última el reconocimiento tridimensional de las estructuras anatómicas y la consecuente consolidación de los conocimientos anatómicos, y su principal desventaja es el olor desagradable, debido a los procesos de fijación. Para la adquisición de habilidades en anatomía clínica se consideraron como las metodologías más adecuadas el estudio de casos, el reconocimiento e identificación de estructuras en material humano fijado y las sesiones de anatomía de superficie, mientras que las que mejor se adaptaban a la adquisición de conocimientos de anatomía descriptiva y funcional eran las clases magistrales y el estudio del material humano fijado. Los métodos peor considerados para estudiar anatomía fueron la utilización de modelos anatómicos plásticos y los recursos informáticos.

Palabras clave. Aprendizaje de la anatomía humana. Material humano fijado. Métodos de enseñanza de la anatomía humana. Resultados del aprendizaje de anatomía.

Methodologies and materials in gross human anatomy learning. Perceptions by digital native medical students

Aim. To know the opinion of the first students generation that has grown up under the information and communication technology about the methodologies currently used in the teaching-learning of gross human anatomy in medical studies.

Subjects and methods. The study was based on the data provided by an anonymously answered survey. Seventy-four students of the second year medical students at the University of Las Palmas de Gran Canaria answered the survey.

Results and conclusions. The methodologies better considered for learning gross human anatomy were the study of cases and the recognition of anatomic structures in fixed human material (prosections and dissection). The students considered the main advantage of the last, the recognition of tridimensional anatomical structures and the consolidation of anatomical knowledge, and the main disadvantage was the unpleasant odour due to the fixing process. Students considered the study of cases, the recognition and identification of structures in fixed human material and the surface anatomy sessions, the methodologies that better suited to acquire skills in clinical anatomy and the lectures and the study of fixed human material the most appropriate methodologies to obtain knowledge about descriptive and functional anatomy. Plastic anatomical models and computing resources were considered the worst methods to study gross human anatomy.

Key words. Anatomy learning outcomes. Fixed human material. Learning human anatomy. Methods of teaching human anatomy.

\section{Introducción}

Dos son los aspectos que obligan a reconsiderar las metodologías a utilizar en el proceso de enseñanza- aprendizaje de la anatomía humana. El primero, las características de la nueva generación de estudiantes y su nueva forma de acceder y procesar la información, y el segundo, las nuevas habilidades que los es-
Departamento de Morfología. Facultad de Ciencias de la Salud. Universidad de Las Palmas de Gran Canaria. Las Palmas de Gran Canaria, España.

Correspondencia:

Dra. Blanca Mompeó Corredera. Departamento de Morfología. Facultad de Ciencias de la Salud. Universidad de Las Palmas de Gran Canaria. Doctor Pasteur, s/n. E-35016 Las Palmas de Gran Canaria.

Fax:

+34928453420

E-mail:

bmompeo@dmor.ulpgc.es

Conflicto de intereses: No declarado.

Competing interests: None declared.

(C) 2014 FEM 
tudiantes de medicina deben desarrollar para afrontar los avances tecnológicos en la medicina clínica.

Nuestros estudiantes actuales han nacido, en su mayoría, durante la última década del siglo $\mathrm{xx} \mathrm{y}$ pertenecen a una generación que Prensky $[1,2]$ ha denominado 'nativos digitales' para diferenciarlos de los 'inmigrantes digitales' o personas que usan las tecnologías de la información y la comunicación, pero no han crecido inmersos en ellas y aunque existe controversia con respecto a esta denominación -debido a que muchos consideran que pertenecer a esta generación no implica necesariamente una buena utilización de los recursos tecnológicos [3]-, todos los autores están de acuerdo en que se trata de un nuevo tipo de estudiantes con unas características y modos de interrelacionarse con el mundo diferentes.

Los 'nativos digitales' o, si preferimos, estudiantes digitales como propone Gallardo-Echenique [3], utilizan las nuevas tecnologías para la práctica totalidad de las actividades de su vida diaria, incluidas las relaciones interpersonales. La forma en que integran sus experiencias les hacen pensar y procesar la información de manera diferente a como lo hacían sus predecesores [1], así que la asunción de que las metodologías de enseñanza que funcionaron para los antiguos estudiantes son válidas para los actuales podría ser errónea. Por otra parte, 'cirugía mínimamente invasiva', 'robótica computarizada', 'resonancia magnética dinámica' y 'telemedicina' son únicamente algunos de los términos del vocabulario de la medicina clínica actual. Ante este escenario, ¿en qué sentido y cómo se ve afectada la enseñanza y el aprendizaje de la anatomía humana?

Actualmente existe controversia entre los anatomistas de todo el mundo sobre las mejores metodologías para enseñar anatomía y sobre si el material humano fijado debe seguir siendo el preferido para el aprendizaje de anatomía humana en los cursos de pregrado.

La disección del cadáver ha sido la metodología preferida y utilizada clásicamente para el aprendizaje de la anatomía, pero ha tenido detractores en las últimas décadas y han sido numerosas las propuestas de utilización de nuevos métodos y materiales para la enseñanza de la disciplina, entre ellos los recursos informáticos. Estos recursos han tenido un gran auge en la educación médica actual. En este contexto, nuestro objetivo fue conocer la opinión de los estudiantes pertenecientes a la generación de 'nativos digitales' acerca de la utilización del material humano fijado y otras metodologías en el proceso de enseñanza-aprendizaje de la anatomía humana.

\section{Sujetos y métodos}

Se realizó una encuesta a estudiantes matriculados en el segundo año de la licenciatura en medicina en el curso académico 2009-2010 en la Facultad de Ciencias de la Salud de la Universidad de Las Palmas de Gran Canaria. Los alumnos cumplimentaron un cuestionario de forma voluntaria y anónima el último día de docencia de la asignatura de 'Anatomía Humana II', según el plan de estudios (1997) de la licenciatura en medicina. La distribución de docencia teórica y práctica en las asignaturas de 'Anatomía Humana I y II' era de un 50\%. La docencia práctica incluía la inspección de prosecciones, disección, utilización de modelos anatómicos plásticos, proyección de videos, recursos informáticos, sesiones de anatomía de superficie, estudio de casos y técnicas de imagen. Por su parte, la evaluación teórica y práctica de los estudiantes consistía en un formato híbrido compuesto por preguntas tipo test de respuesta múltiple y preguntas de respuesta corta junto con una prueba práctica que incluía reconocimiento de las estructuras en el material humano fijado, técnicas de imagen o en las imágenes proyectadas y una evaluación continuada de la actividad de los estudiantes en sus clases prácticas, presentación de trabajos y seminarios.

Un total de 83 cuestionarios fueron entregados por el profesor a los estudiantes durante la última media hora en una clase práctica sin previo aviso. Antes de que los estudiantes comenzaran a responder a las preguntas, se les informó acerca del propósito de la encuesta y los estudiantes que decidieron no participar abandonaron la sala. Finalmente, 74 estudiantes completaron la encuesta.

El cuestionario (Tabla I) se construyó según la siguiente composición: preguntas sobre datos demográficos y preguntas dirigidas a determinar las metodologías y material de estudio preferido por los estudiantes, las ventajas y desventajas de la utilización del material humano fijado y su punto de vista sobre la relación entre las metodologías utilizadas en el proceso de enseñanza-aprendizaje y el desarrollo de habilidades concretas. Cinco de estas preguntas tenían respuesta cerrada y para su valoración se utilizó una escala de respuesta de Likert de 5 puntos (1, irrelevante; 2 , algo relevante; 3 , pertinente; 4 , muy relevante, y 5 , fundamental) para una pregunta y para otras cuatro una posible respuesta (sí/no). Dos fueron preguntas de carácter abierto. Para facilitar el procesamiento y análisis de los datos se utilizó el programa estadístico SPSS. Posteriormente se clasificaron los datos para facilitar su valoración e interpretación. 


\section{Resultados}

La encuesta fue respondida por el $89 \%$ de los estudiantes, $45,5 \%$ varones y $54,5 \%$ mujeres, con una edad media de 19,1 \pm 0,66 años. Los 74 participantes contestaron la totalidad de las preguntas del cuestionario. La mayoría $(85,5 \%$ y $92,4 \%$, respectivamente) consideró que las prácticas con material humano fijado y el estudio de casos eran metodologías fundamentales o muy relevantes en el estudio de la anatomía humana. Las sesiones de anatomía de superficie, técnicas de imagen, clases magistrales y proyección de películas o transparencias fueron consideradas muy relevantes o fundamentales por un $76 \%, 71 \%, 77 \%$ y $71 \%$ de los estudiantes, respectivamente. El uso de modelos anatómicos de plástico y los recursos informáticos fueron considerados muy relevantes o fundamentales por un $38 \%$ y $24 \%$ de los estudiantes, respectivamente (Tabla II).

La tabla III muestra la apreciación de los estudiantes acerca de la relación entre las metodologías de enseñanza utilizadas y las competencias obtenidas.

Para el desarrollo de habilidades y conocimientos de anatomía clínica, las metodologías mejor valoradas fueron el estudio de casos, las prácticas con material humano fijado y las sesiones de anatomía de superficie, consideradas las mas convenientes por el $77,2 \%, 65 \%$ y $42,4 \%$ de los estudiantes, respectivamente. Las clases magistrales, las prácticas con material humano fijado, la utilización de técnicas de imagen, proyecciones de películas y diapositivas, modelos anatómicos de plástico y recursos informáticos fueron considerados convenientes por un $33,3 \%, 19,7 \%, 16,66 \%, 9 \%$ y $9 \%$ de los estudiantes, respectivamente.

Para la adquisición de conocimientos de anatomía descriptiva y funcional, los estudiantes consideran como metodologías adecuadas las prácticas con material humano fijado $(73 \%)$ y las clases magistrales (53\%). La proyección de videos y diapositivas, las técnicas de imagen, el estudio de casos, los modelos anatómicos y los recursos informáticos fueron considerados por un 36,36\%, 18,18\%, 15,15\%, $13,63 \%$ y $12 \%$ del total de los estudiantes participantes, respectivamente.

La tabla IV indica la consideración de los estudiantes sobre las ventajas y desventajas de la utilización del material humano fijado. El 100\% de los estudiantes consideró que la principal ventaja de este tipo de material era favorecer la comprensión tridimensional de las estructuras. El 94\% opinó que el uso de este material hacía el aprendizaje de la anatomía humana más interesante, y el 92\%, que era un buen material para el establecimiento de conoci-
Tabla I. Cuestionario

1. Edad (años)

2. Sexo: varón/mujer

3. Nacionalidad

4. ¿Qué valoración darías a cada una de estos métodos para aprender anatomía en una valoración de $1-5$ (1, irrelevante; 2 , algo relevante; 3, pertinente; 4, muy relevante; 5, fundamental):

a. Estudio con material humano fijado (disecciones o prosecciones)

b. Estudio de casos

c. Sesiones de anatomía de superficie

d. Recursos informáticos

e. Técnicas de imagen

f. Clases magistrales

g. Proyección de transparencias y películas

h. Modelos anatómicos plásticos

5. ¿Qué métodos de enseñanza/aprendizaje consideras más conveniente para adquirir conocimientos de anatomía descriptiva y funcional?

6. ¿Qué métodos de enseñanza/aprendizaje consideras más conveniente para adquirir competencias en anatomía clínica?

7. Considera las siguientes ventajas de la utilización de material humano fijado para la enseñanza/ aprendizaje de la anatomía humana (sí/no):

a. Favorece el reconocimiento tridimensional de las estructuras anatómicas

b. Contribuye a consolidar los conocimientos anatómicos adquiridos previamente

c. Permite a los estudiantes reconocer los efectos producidos por el traumatismo

d. Permite comprender los procedimientos de emergencia

e. Prepara al estudiante para la cirugía.

f. Hace el aprendizaje de la anatomía más interesante.

g. Incrementa el respeto por el cuerpo humano

8. Considera las siguientes desventajas de la utilización de material humano fijado para la enseñanza/ aprendizaje de la anatomía humana (sí/no):

a. Es un trabajo duro

b. A veces es difícil reconocer las estructuras

c. El material huele mal

d. Hace que tenga pensamientos sobre enfermedad o muerte propia

e. Hace que tenga pensamientos sobre enfermedad o muerte de amigos o familiares

f. Es una actividad aburrida

9. El material humano fijado puede ser sustituido por otros métodos de estudio en el proceso de enseñanza/aprendizaje de la anatomía humana (sí/no):

a. Puede ser sustituido por recursos informáticos

b. Puede ser sustituido por modelos anatómicos plásticos

c. Puede ser sustituido por proyecciones de transparencias o películas

d Puede ser sustituido por clases magistrales

e. Puede ser sustituido por libros de texto y atlas

f. No puede ser sustituido por otro tipo de método

10. Señala si estás de acuerdo con las siguientes afirmaciones respecto a la utilización de material humano fijado (sí/no):

a. No hay necesidad de utilizar este tipo de material

b. El estudio con este tipo de material debería ser opcional

c. La utilización de este material no tiene sentido en los estudios de pregrado, debería utilizarse únicamente en posgrado

d. Debería eliminarse de las metodologías de enseñanza de la anatomía humana

e. La utilización de este tipo de material es obsoleta 
Tabla II. Metodologías y materiales utilizados en la enseñanza de la anatomía humana y porcentaje de estudiantes que atribuyeron a cada una de las metodologías una calificación de 405 en su valoración $(n=74)$.

\begin{tabular}{lc}
$\begin{array}{l}\text { Utilización de material humano fijado } \\
\text { para disección o estudio de prosecciones }\end{array}$ & $85,5 \%$ \\
\hline Estudio de casos & $92,4 \%$ \\
\hline Sesiones de anatomía de superficie & $76 \%$ \\
\hline Técnicas de imagen & $71 \%$ \\
\hline Clases magistrales & $77 \%$ \\
\hline Proyección de videos y transparencias & $71 \%$ \\
\hline Modelos anatómicos plásticos & $38 \%$ \\
\hline Recursos informáticos & $24 \%$ \\
\hline
\end{tabular}

mientos previamente adquiridos. Otras consideraciones como la preparación para la cirugía, la comprensión de los procedimientos traumáticos o de emergencia fueron consideradas muy relevantes o fundamentales por el $82 \%, 81 \%$ y $54 \%$ de los estudiantes, respectivamente. Su papel en incrementar el respeto por el cuerpo humano fue considerado por el $60 \%$ de los estudiantes.

En relación con las desventajas del material humano fijado, el $70 \%$ de los estudiantes consideraron que el mal olor era un inconveniente importante. Otras desventajas consideradas fueron la dificultad del reconocimiento de estructuras $(51,5 \%)$ y la aparición de pensamientos acerca de la enfermedad o la muerte (26,6\%). Un $20 \%$ de los estudiantes consideraron difícil la utilización de material fijado y únicamente el $6,1 \%$ consideró que el trabajo con material humano fijado era una actividad aburrida.

La tabla V muestra las respuestas de los estudiantes en relación a la posibilidad de sustitución del material humano fijado por otro tipo de herramienta para el aprendizaje de la anatomía humana. El 18,2\% de los estudiantes consideraron que podría sustituirse por proyección de videos y diapositivas; el 15,2\%, por recursos informáticos; el 10,6\%, por modelos anatómicos plásticos, y el 7,6\%, por clases magistrales, libros o atlas.

La consideración de la totalidad de los estudiantes participantes acerca de la utilidad del material humano en el aprendizaje de anatomía macroscópica se muestra en la tabla VI. El 24,8\% de los estudiantes consideraron que el uso de material humano fijado debería ser opcional, y el 15\%, que debería limitarse a los cursos de posgrado. Sólo el 3\% opinó que este tipo de material debe excluirse de la docencia de la anatomía humana en los estudios de medicina, y el 4,5\%, que es un material obsoleto.

En ningún caso se observó diferencia en función del sexo de los estudiantes.

\section{Discusión}

El planteamiento del trabajo surgió como consecuencia del vertiginoso desarrollo de las tecnologías de la información y la comunicación que, junto a las innovaciones tecnológicas que se han incorporado a la medicina clínica, ha propiciado la aparición de una generación que interrelaciona con el mundo de forma diferente a como lo hacían sus antecesores. Los estudiantes a los que se encuestó tenían una media de 19,1 años, nacieron al inicio de la última década del siglo $\mathrm{xx}$ y, por ello, pueden considerarse 'nativos digitales' [1,2], 'generación Net' [4] o 'millennials' [5]. Se asume que, independientemente de la denominación que se les atribuya, los miembros de esa generación poseen características y habilidades diferentes a las de sus predecesores, distinta forma de pensar y procesar la información y diferentes estilos y preferencias de aprendizaje.

Las metodologías que los encuestados consideraron más relevantes para el estudio de la anatomía humana fueron el estudio de casos y aquellas metodologías que utilizan material humano fijado. Aunque no hemos encontrado en la bibliografía evidencias claras de la efectividad del estudio de casos en la enseñanza de la anatomía, es notorio que motiva a los estudiantes y contribuye a que desarrollen habilidades relacionadas con la aplicación de los conocimientos en situaciones nuevas, favoreciendo el análisis de datos y la reflexión sobre conocimientos previamente adquiridos.

Respecto a la disección y el estudio de prosecciones, los estudiantes le adjudicaron una valoración alta, tanto a nivel general como para la adquisición de conocimientos de anatomía descriptiva y funcional y de competencias en anatomía clínica. Los encuestados consideraron que aquel material les proporciona una concepción tridimensional de las estructuras, favoreciendo la consolidación de los conocimientos y haciendo el aprendizaje más interesante. Tambien opinaron que el trabajo con el material fijado incrementa el respeto por el cuerpo humano, lo que contribuye a la autorreflexión y desarrollo de competencias en el campo del profesionalismo médico. Señalaron como la principal desventaja el olor desagradable. Aspectos emocionales 
Tabla III. Opinión de los estudiantes sobre la relación entre el tipo de metodología y material utilizado en la enseñanza de la anatomía humana y el grupo de competencias adquirido $(n=74)$.

\begin{tabular}{|c|c|c|c|c|c|c|c|c|}
\hline & $\begin{array}{c}\text { Material } \\
\text { humano } \\
\text { fijado }\end{array}$ & $\begin{array}{l}\text { Estudio } \\
\text { de casos }\end{array}$ & $\begin{array}{l}\text { Estudio con } \\
\text { modelos } \\
\text { anatómicos }\end{array}$ & $\begin{array}{c}\text { Sesiones } \\
\text { de anatomía } \\
\text { de superficie }\end{array}$ & $\begin{array}{l}\text { Recursos } \\
\text { informáticos }\end{array}$ & $\begin{array}{l}\text { Técnicas } \\
\text { de imagen }\end{array}$ & $\begin{array}{c}\text { Clases } \\
\text { magistrales }\end{array}$ & $\begin{array}{l}\text { Proyección } \\
\text { de videos y } \\
\text { transparencias }\end{array}$ \\
\hline $\begin{array}{l}\text { Conocimientos de anatomía } \\
\text { descriptiva y funcional }\end{array}$ & $73 \%$ & $15,15 \%$ & $13,63 \%$ & $12 \%$ & $16,6 \%$ & $18,18 \%$ & $53,03 \%$ & $36,6 \%$ \\
\hline
\end{tabular}

como pensamientos sobre la muerte o la enfermedad fueron menos relevante para los estudiantes.

El $76 \%$ de los estudiantes opinaron que el uso del material humano para el aprendizaje de la anatomía no podía sustituirse por otro tipo de material, mientras que aproximadamente el $24 \%$ estimó que podría reemplazarse por otras metodologías. Respecto a este punto, la opinión de la mayoría de los estudiantes fue similar a la de los alumnos de medicina de la misma edad de Reino Unido [6], Australia [7], estudiantes de medicina del último curso de la Universidad de Las Palmas de Gran Canaria hace 10 años [8] y estudiantes de medicina de la Universidad de Southampton [9]. Los resultados son de interés porque aunque no se discute la conveniencia de utilizar material humano fijado para la formación de estudiantes posgraduados, sí se pone en entredicho la necesidad de utilizarlo para la formación de alumnos de pregrado [10-12].

En el proceso de enseñanza-aprendizaje de la anatomía humana se consideran dos tipos de resultados de aprendizaje: la adquisición de los conocimientos básicos anatómicos y el desarrollo de habilidades que permitan la aplicación de los conocimientos adquiridos a la clínica, y aunque estos dos conceptos son interdependientes, constituyen entidades separadas que necesitan para su adquisición diferentes metodologías de enseñanza-aprendizaje. En este sentido, los estudiantes consideraron el estudio de casos, las metodologías que utilizan material humano fijado y las sesiones de anatomía de superficie como las que mejor se adaptan a la obtención de competencias en anatomía clínica, mientras que juzgaron la disección de cadáveres, el estudio de prosecciones y las clases magistrales como las más adecuadas para la obtención de conocimientos básicos de anatomía descriptiva y funcional. Estas opiniones fueron similares a las manifestadas por los estudiantes de segundo curso de medicina de Nottingham y del Imperial College de Londres [6].

Los materiales peor considerados fueron los modelos anatómicos y, curiosamente, los medios in-
Tabla IV. Opinión de los estudiantes sobre las ventajas y desventajas de utilizar el material humano fijado para disección o prosecciones en la enseñanza de la anatomía humana $(n=74)$.

\begin{tabular}{|c|c|c|}
\hline \multirow{7}{*}{ Ventajas } & $\begin{array}{l}\text { Favorece la comprensión tridimensional de } \\
\text { las estructuras anatómicas y sus relaciones }\end{array}$ & $100 \%$ \\
\hline & Consolida los conocimientos anatómicos adquiridos previamente & $92,5 \%$ \\
\hline & $\begin{array}{l}\text { Favorece la comprensión de los procedimientos } \\
\text { diagnósticos y terapéuticos traumáticos }\end{array}$ & $81,0 \%$ \\
\hline & $\begin{array}{l}\text { Favorece la comprensión de los procedimientos } \\
\text { diagnósticos y terapéuticos de emergencias }\end{array}$ & $54,0 \%$ \\
\hline & Hace el aprendizaje de la anatomía humana más interesante & $94,0 \%$ \\
\hline & Prepara para la cirugía & $81,8 \%$ \\
\hline & Incrementa el respeto por el cuerpo humano & $60,6 \%$ \\
\hline \multirow{6}{*}{ Desventajas } & Es una actividad que resulta difícil de realizar & $33,3 \%$ \\
\hline & El reconocimiento de estructuras es difícil & $51,5 \%$ \\
\hline & Mal olor a consecuencia de los procedimientos de fijación & $70,0 \%$ \\
\hline & Aparición de pensamientos sobre la propia muerte o enfermedad & $10,6 \%$ \\
\hline & $\begin{array}{l}\text { Aparición de pensamientos sobre la muerte } \\
\text { o enfermedad de amigos y familiares }\end{array}$ & $16,6 \%$ \\
\hline & Son actividades aburridas & $6,1 \%$ \\
\hline
\end{tabular}

formáticos. Los modelos anatómicos fueron estimados relevantes por un $38 \%$ de los estudiantes. Lo que resulta más sorprendente es que únicamente un $24 \%$ de los estudiantes 'nativos digitales' hayan considerado los recursos informáticos relevantes para el aprendizaje de la anatomía, siendo estas opiniones similares a las de estudiantes de Australia y Reino Unido [7,13]. En la misma línea, Adamczyk et al [14] observaron que únicamente un $30 \%$ de sus estudiantes utilizaban medios informáticos para el estudio de la anatomía y siempre como método com- 
Tabla V. Opinión de los estudiantes en relación a la posible sustitución del material humano fijado por otros materiales o metodologías $(n=74)$.

\begin{tabular}{lc}
\hline Recursos informáticos & $15,2 \%$ \\
\hline Clases magistrales & $7,6 \%$ \\
\hline Modelos anatómicos plásticos & $10,6 \%$ \\
\hline Proyección de videos e imágenes & $18,2 \%$ \\
\hline Atlas y libros de texto & $7,6 \%$ \\
\hline No puede ser sustituido por otro tipo de material & $76,0 \%$
\end{tabular}

plementario, nunca exclusivo en el aprendizaje de esta disciplina.

Friedman [15], en 1994, explicó la resistencia a la utilización de medios informáticos como la consecuencia del efecto inhibidor de las tecnologías sobre usuarios novatos, pero esta explicación resulta poco probable para los estudiantes nacidos a partir de 1990. Sin embargo, podría considerarse la posibilidad de que aunque esta generación esté familiarizada con el uso de las tecnologías y sus habilidades digitales sean elevadas, su capacidad de evaluar y aprender desde los medios digitales podría estar limitada [3]. Otras razones que podrían considerarse para el escaso arraigo de los recursos informáticos en el aprendizaje de la anatomía humana serían una utilización poco convincente de los recursos por el profesor, que es un 'inmigrante digital', o la propia naturaleza de la disciplina de anatomía humana, que determinaría la metodología más adecuada para su enseñanza. De hecho, los trabajos que han evaluado los resultados de la utilización de medios informáticos en la enseñanza de la anatomía humana no han podido demostrar que sean, por sí solos, eficaces para mejorar los resultados de los estudiantes, aunque sí han demostrado su eficacia en combinación con otras metodologías [16-18]. En este sentido, debe tenerse en cuenta que los recursos informáticos incluyen diferentes aspectos y que sería necesario el análisis individualizado de cada uno de ellos para determinar cuáles y bajo qué circunstancias pueden ser útiles en el aprendizaje de la anatomía humana.

En conclusión, los estudiantes 'nativos digitales' encuestados consideraron adecuada la utilización del material humano fijado para la enseñanza/ aprendizaje de la anatomía humana en los estudios de medicina y que el empleo de los recursos informáticos no parece tener, por el momento, una aceptación mayoritaria para el estudio de la disciplina.
Tabla VI. Consideraciones sobre la necesidad de utilizar material humano fijado $(n=74)$.

\begin{tabular}{|c|c|}
\hline No hay necesidad de utilizar este tipo de material & $6,1 \%$ \\
\hline El estudio con este tipo de material debería ser opcional & $24,8 \%$ \\
\hline $\begin{array}{l}\text { La utilización de este material no tiene sentido en los estudios } \\
\text { de pregrado, debería utilizarse únicamente en posgrado }\end{array}$ & $15,2 \%$ \\
\hline $\begin{array}{l}\text { Debería eliminarse de las metodologías } \\
\text { de enseñanza de la anatomía humana }\end{array}$ & $3,0 \%$ \\
\hline La utilización de este tipo de material es obsoleta & $4,5 \%$ \\
\hline
\end{tabular}

Bibliografía

1. Prensky M. Digital natives, digital immigrants. In Prensky M. On the horizon. UK: MCB University Press; 2001. vol. 9, n. ${ }^{\circ} 5$

2. Prensky $M$. The emerging online life of the digital native. 2004. URL: http://www.marcprensky.com/writing/prensky. [10.12.2012].

3. Gallardo-Echenique EE. Hablemos de estudiantes digitales y no de nativos digitales. UT. Revista de Ciències de l'Educació 2012; 7-21.

4. Tapscott D. Educating the net generation. Educ Leadersh 1999; 56: 6-11.

5. Howe N, Strauss W. Millennials rising: the next greatest generation. New York: Vintage Books; 2000.

6. Kerby J, Shukur ZN, Shalhoub J. The relationships between learning outcomes and methods of teaching anatomy as perceived by medical students. Clin Anat 2011; 24: 489-97.

7. Azer SA, Eizenberg N. Do we need dissection in an integrated problem-based learning medical course. Perceptions of first and second-year students. Surg Radiol Anat 2007; 29: 173-80.

8. Mompeó B, Pérez L. Relevancia de la anatomía humana en el ejercicio de la medicina de asistencia primaria y en el estudio de las asignaturas de segundo ciclo de la licenciatura en Medicina. Educ Med 2003; 6: 41-51.

9. Smith CF, Mathias HC. What impact does anatomy education have on clinical practice? Clin Anat 2011; 24: 113-9.

10. McLachlan JC. New path for teaching anatomy: living anatomy and medical imaging vs. dissection. Anat Rec 2004; 281: 4-5.

11. McLachlan JC. Patten D. Anatomy teaching: ghosts of the past, present and future. Med Educ 2006; 40: 243-53.

12. Rizzolo LJ, Steward WB. Should we continue teaching anatomy by dissection when...? Anat Rec 2006; 289: 215-8.

13. Moxham BJ, Moxham SA. The relationships between attitudes, course, aims and teaching methods for the teaching of gross anatomy in medical curriculum. Eur J Anat 2007; 11 (Supl 1): 19-30.

14. Adamczyk C, Holzer M, Putz R, Fischer MR. Student learning preferences and the impact of a multimedia learning tool in the dissection course at the University of Munich. Ann Anat 2009; 191: 339-48.

15. Friedman CP. The research we should be doing. Acad Med 1994; 69: 455-7.

16. Biassuto SN, Caussa LI, Criado del Río LE. Teaching anatomy: cadavers vs. computers? Ann Anat 2006; 188: 187-90.

17. McNulty JA, Halama J, Espiritu B. Evaluation of computeraided instruction in the medical gross anatomy curriculum. Clin Anat 2004; 17: 73-8.

18. Levine MG, Stempak J, Conyers G, Walters JA. Implementing and integrating computer-based activities into a problem based gross anatomy curriculum. Clin Anat 1999; 12: 191-8. 